López López, M.C.; León Guerrero, M.J. y Pérez García, P. (2018). El enfoque por competencias en el contex̄to universitario español. La visión del profesorado. Revista de Investigación Educativa, 36(2), 529-545.

DOI: http://dx.doi.org/10.6018/rie.36.2.314351

\title{
El enfoque por competencias en el contexto universitario español. La visión del profesorado'
}

\author{
The Competency-Based Approach in the Spanish University \\ Context. The Vision of the Teaching Staff
}

\author{
María del Carmen López López, María José León Guerrero y Purificación Pérez García \\ Didáctica y Organización Escolar. Facultad de Ciencias de la Educación. Universidad de Granada (España)
}

\begin{abstract}
Resumen
La adopción del enfoque basado en competencias comporta un cambio importante desde el punto de vista pedagógico y didáctico que afecta directamente a la formación universitaria y a la práctica docente. Sin embargo, los esfuerzos institucionales desplegados para favorecer los cambios estructurales han sido superiores a la atención deparada al cambio cultural y pedagógico que afecta al profesorado. Por esta razón, y con el propósito de ampliar los espacios de reflexión y valorar los progresos alcanzados en estos últimos años, hemos profundizado en las percepciones que tienen los docentes de distintas universidades españolas sobre el enfoque por competencias. El estudio, de corte cualitativo, se ha basado en grupos de discusión como técnica de recogida de datos. En él han participado 43 profesores de seis universidades espanolas públicas. La información resultante de las grabaciones ha sido transcrita y categorizada siguiendo un proceso deductivo-inductivo. La información proporcionada ha sido estructurada en torno a 36 subcategorías, agrupadas en 5 categorías. El análisis de resultados permite
\end{abstract}

Correspondencia: María del Carmen López López. Departamento de Didáctica y Organización Escolar. Facultad de Ciencias de la Educación. Universidad de Granada. Campus Universitario de Cartuja S/N. 18071, Granada. Correo electrónico: mclopez@ugr.es

1 Investigación financiada por el Ministerio de Economía y Competitividad de España (Ref. EDU201232766), titulada: "El impacto de la evaluación educativa en el desarrollo de competencias en la universidad. La perspectiva de las primeras promociones de graduados". 
concluir que el profesorado universitario tiene dificultades para incorporar las competencias transversales al currículum académico, tiende a mostrarse pesimista respecto al impacto del enfoque por competencias en la mejora de la práctica educativa y considera que la incorporación exitosa de este enfoque pasa por profundizar en la actual reforma educativa, adoptar perspectivas más holísticas y atender a los aspectos culturales del cambio.

Palabras clave: profesorado; educación superior; competencias; percepciones.

\begin{abstract}
The adoption of the competency-based approach means an important change from the pedagogic and didactic point of view that directly affects university education and teaching practice. However, there is a certain tendency to admit that the institutional efforts made to stimulate structural changes have been superior to the attention given to the pedagogical and cultural change that affect teachers. For this reason and with the aim to expand the areas of critical thinking and value the improvement achieved in recent years, and to make visible the complications detected, our purpose is to delve into the perceptions that teachers of different Spanish universities have about the competency-based approach. The qualitative study was based on discussion groups as a data collection technique. Forty-three professors from six public Spanish universities had participated in the study. The recorded information given has been transcribed and categorized following a deductive-inductive process. The information has been structured around thirty-six subcategories and grouped in five categories. The analysis of the results concluded that the university professors have difficulties with incorporating the transversal competencies into the academic curriculum. They tend to be pessimistic regarding the impact of the competency-based approach in improving educational practice and consider that the successful incorporation of this approach requires deepening the current educational reform, adopting more holistic perspectives and addressing the cultural aspects of change.

Keywords: teachers; word; competency; higher education; perceptions.
\end{abstract}

\title{
Introducción
}

En los últimos años la universidad española ha experimentado un proceso de transformación como respuesta a los compromisos adquiridos con la creación del Espacio Europeo de Educación Superior (EEES). El Proceso de Bolonia no sólo ha abierto el debate público en torno al sentido y finalidad de la institución universitaria en el siglo XXI, sino que ha invitado a redefinir su compromiso con el entorno. Convertir la universidad en motor de desarrollo económico en la era del conocimiento, mejorando su conexión con el mercado laboral y la empleabilidad de los egresados, se ha convertido en un tema recurrente de la política educativa universitaria en los últimos tiempos en el ámbito nacional e internacional (Pozo \& Bretones, 2015; Watson, 2011).

Las actuaciones emprendidas en el contexto español han sido numerosas y de distinta índole, pues junto a los esfuerzos para promover los cambios normativos y estructurales, necesarios para favorecer la apertura de la universidad al ámbito laboral, su internacionalización y la adaptación de sus titulaciones al marco europeo, también se han impulsado iniciativas dirigidas a facilitar la adopción de un nuevo enfoque 
educativo centrado en el aprendizaje de competencias (Medina, Domínguez \& Sánchez, 2015; Navarro, González, López \& Botella, 2015). La adopción de este enfoque comporta un cambio importante desde el punto de vista pedagógico y didáctico que afecta a la formación universitaria y a la práctica docente. Con él se pretende poner en marcha programas educativos sustentados en perfiles profesionales, revalorizar la formación práctica, impulsar nuevos roles docentes centrados en la orientación y seguimiento del aprendizaje, introducir metodologías activas que favorezcan el papel activo del alumnado en su formación, incorporar nuevos sistemas de evaluación, etc. Cambios que representan un desafío para la cultura institucional y profesional arraigada en la universidad española.

En estos momentos en que asistimos a la graduación de las primeras promociones de estudiantes en las titulaciones diseñadas conforme al EEES, resulta de vital importancia emprender estudios que permitan analizar el impacto real de las acciones emprendidas, así como las dificultades encontradas, y establecer, a partir de ellos, nuevos compromisos para la mejora. Existe, en este sentido, una cierta tendencia a reconocer que los esfuerzos institucionales desplegados para favorecer los cambios estructurales han sido superiores a la atención deparada a los aspectos culturales, didácticos y pedagógico que estos cambios comportan para el profesorado (López, Benedito \& León, 2016). La mejora de la formación práctica, la formación y coordinación docente, la evaluación de competencias, la innovación metodológica, o la situación profesional de los docentes, continúan siendo asignaturas pendientes en el sistema universitario español (Pozo \& Bretones, 2015). Para estas autoras, gran parte de las dificultades que se apreciaban en un primer momento siguen sin solucionarse, opinión que comparten Ermenc \& Vujisic-Zivkovic (2015) cuando afirman que aún estamos lejos de conseguir que la incorporación del enfoque por competencias sea un éxito.

Aunque existen trabajos recientes que persiguen subsanar algunas de estas deficiencias, los resultados no son concluyentes (Miguel de \& Lázaro de, 2016; Villa, Arranz, Campo \& Villa, 2015). Ermenc \& Vujisic-Živkovic (2015) y Watson (2011) reconocen que la información que tenemos, para determinar el verdadero alcance de los cambios adoptados y su incidencia en la mejora de la práctica formativa, es insuficiente y recomiendan ampliar la investigación y profundizar en el enfoque por competencias y su impacto.

Para Cabrera, López y Portillo (2016), Euler (2015) y Tran (2013), responder a la reforma promovida desde Bolonia supone hacer frente a numerosos retos que tienen que ver, entre otras cuestiones, con el desarrollo organizativo de las estructuras, la gestión del cambio, el tipo de liderazgo, el plan de estudios, las culturas universitarias, la formación docente, las condiciones laborales y los sistemas de evaluación de la calidad docente. Comprobamos, no obstante, que entre los numerosos factores que suelen destacarse siempre hay uno que figura de manera reiterada: el profesorado. Los docentes constituyen un factor clave para el éxito en cualquier proceso de mejora educativa (Hallinger, Heck \& Murphy, 2014).

Por esta razón, hemos profundizado en las percepciones que tienen los docentes de distintas universidades españolas sobre el enfoque por competencias, los obstáculos encontrados en su incorporación y el impacto que, en su opinión, han tenido las acciones emprendidas en estos últimos años. 


\section{Método}

\section{Objetivos}

La finalidad de este trabajo es estudiar las opiniones del profesorado universitario acerca del enfoque por competencias. Esta finalidad se concreta en los siguientes objetivos:

1. Conocer las percepciones del profesorado universitario acerca del enfoque por competencias como opción formativa en la educación superior.

2. Identificar los obstáculos que, en opinión del profesorado, tiene la incorporación del enfoque por competencias a la formación universitaria.

3. Analizar el impacto del enfoque por competencias en la formación universitaria.

\section{Enfoque metodológico}

Para responder a la finalidad del estudio se ha optado por un enfoque de naturaleza cualitativa e interpretativa. Este enfoque permite profundizar en las percepciones desde la mirada de las personas implicadas, utilizando sus propias palabras (Gibbs, 2012).

\section{Población y Muestra}

En el estudio han participado 43 docentes pertenecientes a las seis universidades públicas españolas que participan en el proyecto de investigación en que se enmarca este trabajo: Universidad Rovira y Virgili (URV), Universidad Autónoma de Barcelona (UAB), Universidad de Barcelona (UB), Universidad de las Islas Baleares (UIB), Universidad de Granada (UGR) y Universidad Oberta de Catalunya (UOC).

Se ha seguido un muestreo intencional (Albert, 2006) y para la selección de los componentes de los grupos de discusión se han aplicado los siguientes criterios heterogéneos (Callejo, 2001): docentes que imparten docencia en distintos centros, grados y tipos de materias (obligatorias y optativas); pertenecientes a diferentes departamentos y áreas de conocimiento; hombres y mujeres; noveles y experimentados; y de distinta edad. Los grupos de discusión se definieron gracias a la mediación de los coordinadores de las titulaciones.

Finalmente, se conformaron seis grupos de discusión (uno por universidad) integrados por 4 a 10 miembros, con edades comprendidas entre los 29 y 62 años, 24 son mujeres y 19 hombres, con experiencia docente que oscila entre 5 y 36 años.

\section{Procedimiento de recogida y análisis de información}

El estudio utiliza los grupos de discusión como técnica de recogida de datos, ya que se considera una buena opción para profundizar en las opiniones/percepciones del profesorado universitario acerca del enfoque por competencias (Callejo, 2001).

En la Tabla 1 se presenta la configuración de los grupos de discusión que han participado en la investigación por universidades, centros y departamentos. 
Tabla 1

Distribución de grupos de discusión por universidad, centros y departamentos

\begin{tabular}{|c|c|c|}
\hline Universidad & Facultad & Departamentos \\
\hline URV & $\begin{array}{l}\text { Escuela Técnica Superior de Ingeniería } \\
\text { Facultad de Ciencias de la Educación y } \\
\text { Psicología }\end{array}$ & $\begin{array}{l}\text { Ingeniería Informática Matemáticas } \\
\text { Pedagogía }\end{array}$ \\
\hline $\mathrm{UAB}$ & Escuela Universitaria Salesiana Sarrià & $\begin{array}{l}\text { Formación básica } \\
\text { Electrónica } \\
\text { Educación musical, plástica y corporal } \\
\text { Didáctica de la lengua y la literatura } \\
\text { Mecánica }\end{array}$ \\
\hline UB & $\begin{array}{l}\text { Farmacia } \\
\text { Formación del Profesorado }\end{array}$ & $\begin{array}{l}\text { Fisicoquímica } \\
\text { Tecnología farmacéutica } \\
\text { Didáctica expresión musical y corporal } \\
\text { Didáctica y organización educativa } \\
\text { Didáctica de la lengua y la literatura }\end{array}$ \\
\hline UIB & $\begin{array}{l}\text { Escuela Politécnica Superior } \\
\text { Ciencias }\end{array}$ & $\begin{array}{l}\text { Matemáticas } \\
\text { Informática } \\
\text { Física }\end{array}$ \\
\hline UGR & $\begin{array}{l}\text { Farmacia } \\
\text { Ciencias de la Educación }\end{array}$ & $\begin{array}{l}\text { Bioquímica y biología molecular } \\
\text { Fisiología vegetal } \\
\text { Farmacia y tecnología farmacéutica } \\
\text { Historia, legislación y gestión } \\
\text { Métodos de investigación y diagnósti- } \\
\text { co en educación } \\
\text { Didáctica y organización escolar }\end{array}$ \\
\hline UOC & $\begin{array}{l}\text { Estudios informáticos, multimedia y } \\
\text { telecomunicaciones }\end{array}$ & \\
\hline
\end{tabular}

El procedimiento organizativo seguido para la realización de las entrevistas grupales ha sido el siguiente: se convocó a los componentes del grupo de discusión por correo electrónico, teléfono o personalmente. Una vez reunidos, se comunicó a los asistentes la finalidad de la investigación, se enfatizó su protagonismo como conocedores "directos" de la temática que se iba a desarrollar, y se registró la discusión mediante grabadora. Las sesiones tuvieron una duración aproximada de dos horas cada una.

La discusión fue dinamizada por la misma moderadora en todos los grupos, lo cual supuso su desplazamiento a todas las universidades. Los temas tratados giraron en torno a las competencias como contenido y elemento en torno al cual se estructuran las titulaciones, su contribución al aprendizaje, su incorporación a la práctica, su repercusión (en centros, profesorado, alumnado, práctica...). También se incluyó la posibilidad de introducir otras cuestiones vinculadas al tema que no hubieran sido tratadas. 
La información resultante ha sido transcrita y categorizada siguiendo un proceso deductivo-inductivo (Strauss \& Corbin, 2002). En primer lugar, se partió de dimensiones generales obtenidas tras la revisión del marco teórico referencial. En segundo lugar, se procedió a hacer una lectura reflexiva de las transcripciones y se fueron extrayendo temas surgidos de las opiniones de los docentes. El siguiente paso supuso, como aconsejan los anteriores autores, cotejar las dimensiones y los temas extraídos para volver a releer los datos y perfilar un sistema de categorías que agrupara las características de las percepciones de los participantes. El análisis se realizó a partir de la categorización por redes sistémicas, siguiendo el método de comparación constante (Glaser \& Strauss, 1967).

El sistema resultante estuvo compuesto por 36 subcategorías, agrupadas en 5 categorías. El proceso de análisis de la información no precisó de ningún software cualitativo, pues el volumen de datos permitió que se hiciera de forma manual.

\section{Resultados}

En la Tabla 2 presentamos las categorías y subcategorías extraídas del análisis de la información proporcionada por los docentes universitarios en los distintos grupos de discusión y sus frecuencias.

La información proporcionada por el profesorado universitario sobre el enfoque por competencias ha sido estructurada en torno a cinco categorías. La más recurrida ha sido la relativa a las competencias transversales, seguida de dificultades para su adopción e impacto. Las categorías con las frecuencias más bajas corresponden a: valoración del enfoque por competencias y características de las competencias.

Si tomamos de referencia esta última categoría, encontramos que entre las características más destacas de las competencias figuran: su vinculación al contexto profesional y su conexión con la acción y, en menor medida, su carácter integrador y los diferentes niveles de logro que comporta.

En relación a la trasmisión de competencias, yo veo que van más allá de transmitir conocimientos. Sí que es fundamental que los alumnos vean la aplicación que tienen estos conocimientos en su futuro profesional (P7.UGR)

Yo creo que el asunto es situar la materia en el ejercicio de la profesión, en el rincón que encontremos. Esto ayuda a hacer algo a favor de la competencia aunque sea una asignatura conceptual (P2.UAB)

En cuanto a la valoración del enfoque por competencias, las posiciones de los docentes universitarios que participan en la investigación están divididas, de manera equilibrada, entre aquellos que tienen una visión positiva y los que manifiestan abiertamente su rechazo. Entre las cuestiones que valoran positivamente figuran: los cambios estructurales, las posibilidades de innovación y renovación que ofrece, la apertura a nuevos entornos formativos y el carácter prescriptivo conferido a las competencias en los planes de formación y guías docentes. 
Tabla 2

Categorías, subcategorías y frecuencias

\begin{tabular}{|c|c|c|}
\hline Categoría & Subcategorías & Frecuencia \\
\hline \multirow{4}{*}{$\begin{array}{l}\text { Características } \\
(24)\end{array}$} & Implica distintos niveles de logro & 2 \\
\hline & Carácter integrador & 4 \\
\hline & Ligadas a la acción "aplicación" & 7 \\
\hline & Vinculadas al contexto profesional & 11 \\
\hline \multirow{5}{*}{$\begin{array}{l}\text { Valoración } \\
(41)\end{array}$} & Visión positiva del cambio & 11 \\
\hline & Rechazo & 10 \\
\hline & Valoración de las competencias en general & 7 \\
\hline & Valoración de las competencias transversales & 10 \\
\hline & Valoración de las competencias específicas & 3 \\
\hline \multirow{13}{*}{$\begin{array}{l}\text { C. Transversales } \\
(86)\end{array}$} & Trabajo en grupo & 18 \\
\hline & Expresión oral-escrita y habilidades comunicativas & 18 \\
\hline & Planificar & 4 \\
\hline & Compromiso social & 1 \\
\hline & Aprender a aprender & 5 \\
\hline & Reflexión & 8 \\
\hline & Responsabilidad & 3 \\
\hline & Creatividad y emprendimiento & 5 \\
\hline & Uso de TICS y gestión de información & 4 \\
\hline & Gestión de conflictos & 3 \\
\hline & Aplicar teoría a práctica & 11 \\
\hline & Liderazgo & 4 \\
\hline & Dominio Lengua extranjera & 2 \\
\hline \multirow{7}{*}{$\begin{array}{l}\text { Dificultades para } \\
\text { su adopción } \\
\text { (75) }\end{array}$} & Evaluar competencias en un contexto académico & 6 \\
\hline & Falta de apoyo y recursos & 19 \\
\hline & Carácter impositivo y prescriptivo de las competencias & 10 \\
\hline & Falta formación docente & 22 \\
\hline & No desarrolladas en niveles educativos anteriores & 3 \\
\hline & Individualismo estudiantes y falta de compromiso & 6 \\
\hline & Falta coordinación & 9 \\
\hline \multirow{7}{*}{$\begin{array}{l}\text { Impacto } \\
(66)\end{array}$} & No hay cambios en la práctica formativa & 38 \\
\hline & Expresión oral-escrita & 4 \\
\hline & Mejora la retroalimentación. & 8 \\
\hline & Mejora resultados académicos & 2 \\
\hline & Incremento de la participación y trabajo en grupo & 2 \\
\hline & Aumento de la carga de trabajo a los estudiantes & 5 \\
\hline & En el trabajo docente & 7 \\
\hline
\end{tabular}


En la implementación de los grados en sí, sí que creo que, por lo menos en nuestro centro y en nuestros grados, ha habido un cambio estructural que ha propiciado que podamos promover un desarrollo de las competencias (P8.UGR)

... encontré la oportunidad de empezar de nuevo. Era una materia que creo que no se había hecho nunca antes en la universidad, con un grupo nuevo, yo nunca había hecho clases en la politécnica, y toda una estructura nueva. Entonces, directamente pude diseñar este curso desde cero y a partir de competencias (P10.UIB)

Sí que a veces el hecho de hacer otras actividades fuera de la universidad les ayuda a tener estas competencias que le servirán ya para cuando realicen su trabajo profesional (P2.URV).

Los motivos por los que los docentes del estudio rechazan el enfoque por competencias son de distinta índole: falta de recursos y apoyos, la homogenización a la que se tiende y la devaluación de los contenidos.

¿Dónde están los recursos económicos? Esto no es cuestión de buena voluntad. Es por no tener dinero encima. Contrate profesorado y ya verá usted cómo saldremos de las clases magistrales. Que no lo dejen todo a la buena voluntad del profesorado, que la tenemos (P5.UGR).

Unos estudios donde todo vale igual para todo el mundo. Realmente están haciendo aquello de querer hacer a todo el mundo igual. (P1.UIB)

... lo que no puedes hacer es estar bajando los contenidos. Si es importante y resulta que la universidad tiene que hacer alguna cosa, se pone una materia nueva donde se trate este tema (P3.UIB).

En general, consideran que el enfoque por competencias comporta un cambio de mentalidad, en el profesorado y el alumnado, que debe proyectarse en una nueva práctica formativa y esto, en su opinión, no resulta fácil.

no sólo está en el profesorado. Creo que también está en el alumnado. Por eso que comentaba: tenemos implícitos estos valores tan tradicionales que cambiarlos supone cambiar nuestro propio esquema de valoración de lo que necesitamos. Sino realmente estaremos siempre haciendo lo mismo con distinto nombre (P6.UGR)

Gran parte de las aportaciones del profesorado han aludido a las competencias transversales, que han sido valoradas 10 veces y referenciadas en 86 ocasiones. En opinión del profesorado el reto está, fundamentalmente, en el desarrollo de este tipo de competencias. El cambio significativo son las transversales (P4.UOC). En cambio, la frecuencia en que se alude a las competencias específicas es significativamente menor (3), quizá porque, como ellos mismos afirman, las competencias específicas siempre han estado, más o menos explícitamente, en la enseñanza tradicional. A nivel de competencias específicas no veo una diferencia (P4.UOC).

Consideran, en este sentido, que, aunque se ha avanzado en el desarrollo de las competencias transversales (En otras competencias más transversales sí que creo que hemos mejorado bastante (P9.UIB), aún no se han abordado adecuadamente. 
Si todo eso (en referencia a las competencias transversales) se formara también en la universidad, ese salto que tenemos en este país entre la empresa, la universidad y el mundo laboral, que es un auténtico tabú, sería menor para el alumnado, para la empresa y también para nosotros. (P8.UGR)

Entre las competencias transversales más recurridas por los docentes universitarios figuran: el trabajo en equipo, expresión oral-escrita y habilidades comunicativas y aplicación de la teoría a la práctica.

todo esto lo hacemos a través del trabajo en equipo porque es una competencia valorada para incorporar (P3.UOC)

También tienen que saber escribir y redactar informes que aquí también encontramos un punto débil (P7.UOC)

Las competencias transversales menos aludidas son: compromiso social y dominio de una lengua extranjera.

Si nos centramos en la categoría relativa a las dificultades que comporta la adopción del enfoque por competencias, el profesorado considera que el mayor contratiempo es la falta de formación y apoyo institucional al profesorado, el carácter prescriptivo de las competencias, la falta de coordinación y, en menor medida, la evaluación de competencias, el individualismo, la falta de compromiso del alumnado y el hecho de que la formación en competencias no se aborde en otros niveles educativos no universitarios.

A lo largo de las discusiones, los docentes han reconocido, de manera reiterada, su escasa preparación para llevar a cabo la formación por competencias, así como la falta de apoyo institucional que han recibido.

$Y$ en estos treinta y muchos años de docencia, pues uno ha ido improvisando y me temo que, con el nuevo procedimiento de enseñar transmitiendo competencias, pues estamos de nuevo improvisando. Nadie nos ha enseñado ni nos ha sugerido el cómo se debe hacer esta transmisión de competencias (P5.UGR)

Pero yo creo que tenemos un problema sobre las competencias: no sabemos, es voluntarismo todo, y no está reconocido por los centros ni evidentemente por la universidad. Lo veo muy lejos (P5.UB)

Esta falta de formación docente y el escaso apoyo proporcionado por la institución universitaria dificultan un desempeño profesional eficaz y satisfactorio que, como han reconocido los propios docentes, repercute en la actitud del profesorado, en su práctica y en los resultados de aprendizaje.

Soy un poco escéptica con este modelo por competencias. Quizás lo que me pasa es que me falta formación (P3.UOC)

Simplemente he intentado que la evaluación reflejase aquellas competencias que eran importantes. Pero no las he conseguido mejorar ni lo voy a conseguir. No se me ocurre nada (P5.UIB). 
Consideran, así mismo, que sería necesario intensificar y extender la colaboración y coordinación de las acciones formativas dentro y fuera de la universidad.

No solo estrategias colaborativas en el aula, sino ellos como profesionales tienen que coordinarse con los otros profesionales (P1.URV)

Por otra parte, el carácter prescriptivo conferido a las competencias en los planes de formación y en las guías docentes parece generar cierto malestar entre el profesorado que termina considerándolo un obstáculo para la innovación y mejora del proceso enseñanza-aprendizaje.

Para mí, la realidad es esta: que las competencias son códigos que están impuestos, que alguien decidió que eran esos, y ya está. Y se copian y se pegan. (P8.UIB)

El establecer guías que nosotros lo hemos tomado como un contrato con el alumno. Entonces, nos están encorsetando y no nos dejan creatividad en clase. Es decir, si a mí se me ocurre hacer algo distinto a lo que dicen las guías o el tema de la evaluación, por innovar, no lo puedo hacer porque en la guía no está establecido. (P1.UGR)

Los docentes universitarios inciden en la dificultad que entraña la evaluación de competencias en el ámbito académico. Consideran que el contexto universitario no permite evaluar si el alumno posee o no ciertas competencias, ni el grado en que las ha adquirido porque se trabaja sobre situaciones que no son reales. En su opinión, la evaluación por competencias debe realizarse en el ámbito profesional.

... resolver situaciones complejas en un contexto determinado, yo planteo ¿cómo podemos evaluar competencias que se tendrán que desarrollar en un contexto profesional, cuando no están en un contexto profesional? Quizás les podemos ir orientando sobre las competencias que aplicará después; pero no sé si en el contexto universitario podemos evaluar todas las competencias (P8.UB)

Los profesores consideran también que el individualismo y la falta de implicación del alumnado en su aprendizaje representan serios obstáculos para el desarrollo de competencias en el ámbito universitario.

algunos de los problemas que veo es que tienen fama de trabajar mucho de manera individualista (P4.URV)

$Y$ tienes sorpresas, a veces, de alumnos que no se implican y luego los ves fuera muy implicados..., aquí, en clase, están bloqueados con tanto trabajo (P2.URV)

Finalmente, en relación al impacto del enfoque por competencias, encontramos que las alusiones más frecuentes inciden en que la repercusión en la práctica formativa ha sido nula, seguidas de aquellas otras que subrayan los efectos positivos, las que reconocen que el impacto en el trabajo docente ha sido desigual y, por último, las que subrayan un incremento en la carga de trabajo del alumnado. 
Son, por tanto, muy frecuentes, los comentarios que inciden en que la práctica formativa no ha cambiado con la incorporación del enfoque por competencias.

Y estamos dando la misma información, con el mismo método. Y las funciones que van a realizar son las mismas. Por lo menos en nuestro grado, no hay ningún cambio, por lo tanto, desde nuestro punto de vista no supone cambiar nada (P4.UGR)

Este impacto nulo se debe, como reconocen algunos docentes, al hecho de que ya trabajaban con metodologías activas y desarrollaban competencias antes de la creación del Espacio Europeo de Educación Superior, aunque también asumen que hay docentes que no lo hacían antes, ni ahora tampoco.

Y también estoy muy de acuerdo en que había mucha gente que ya hacía esto antes de que a alguien se le ocurriera cambiar los planes de estudio y entra dentro de este Espacio Europeo de Educación Superior. Es más, yo diría que la gente que ya intentaba hacer cosas antes, metodologías orientada a proyectos, lo seguimos haciendo. $Y$ la gente que no lo hacía continúa sin hacerlo. (P1.UIB)

Ahora hablamos de competencias y antes hablábamos de objetivos de aprendizaje, pero en el fondo nuestros estudiantes han de saber lo mismo." (P2.UOC)

Son menos las referencias en las que se asume que ha mejorado la formación o algunos aspectos de la misma. Concretamente, los docentes reconocen que se ha mejorado la retroalimentación ofrecida al alumnado, la expresión oral-escrita, la participación de los estudiantes y el trabajo en grupo, y los resultados académicos.

Allí se resuelve, lo escriben, me lo entregan y lo corrijo para el próximo día que lo tengan. Y esto es el efecto de mejora que yo veo. Que no me lo ha dicho nadie. Que es muchísimo trabajo. He llegado a tener 72 estudiantes, es decir, mucho trabajo. Pero me da igual. Yo creo que así aprenden. (P10.UIB)

Todo esto sí que antes no se hacía para nada y ahora sí que les hacen hacer problemas en grupo, fomentar mucha más participación en clase (no como antes, que yo les hacía los problemas a la pizarra). Ahora no, ahora ellos tienen que salir a la pizarra (P9.UIB) Entonces sí que es cierto que hemos hecho avances importantes, ha aumentado la tasa de éxito en esta asignatura y los estudiantes tienen un alto grado de satisfacción (P5.UB)

Sin embargo, el profesorado del estudio considera que el enfoque por competencias, tal y como se está implementando, ha supuesto un aumento del trabajo para los estudiantes.

Porque también es verdad que tienen una carga de trabajo horrible que no controla nadie. Yo las primeras semanas tengo la clase hasta las últimas filas y entonces empiezo a ver las filas vacías porque tienen una presentación o cualquier otra cosa. Tienen cantidad de trabajos. ¿Por qué tienen que hacer tantos trabajos? ¿Por qué los tienen que hacer todos, si desde bachillerato y la ESO que están haciendo trabajos? Más vale pocas cosas y bien focalizadas (P5.UB) 


\section{Discusión y conclusiones}

Los profesores que participan en el estudio destacan, como cualidades de las competencias, su vinculación con la acción, su conexión con el contexto profesional y su carácter integrador (conocimientos, habilidades, actitudes...). Reconocen que la incorporación de las competencias a la formación universitaria puede facilitar a los estudiantes la aplicación de la teoría a la práctica y un mejor desempeño profesional en el contexto laboral. Aunque las críticas vertidas sobre las competencias durante el proceso de convergencia europea han sido numerosas y las reticencias a su incorporación manifiestas, fundamentalmente por su cercanía al pensamiento conductista y a la perspectiva técnica de la enseñanza (Lozano, Boni, Peris \& Hueso, 2012), las concepciones expuestas por los docentes en esta investigación tienden a reconocer que las competencias deben ser abordadas desde una perspectiva más holística que técnica. Esta consideración les lleva a destacar su potencial formativo en la aplicación del conocimiento a situaciones concretas y su contribución a una mejor preparación de los estudiantes para hacer frente a los desafíos de la práctica profesional, tal y como han reconocido Ermenc \& Vujisic-Živkovic (2015, p. 105): "incluso los críticos más severos del concepto de competencia expresan la postura de que el enfoque de competencia -si se entiende holísticamente y se utiliza con sabiduría- podría influir positivamente en la capacidad de los estudiantes para utilizar conocimientos teóricos y habilidades de investigación cuando se enfrentan a desafíos profesionales complejos".

Los docentes del estudio valoran positivamente, sobre todo, los cambios estructurales realizados por la institución universitaria, la apertura a nuevos entornos formativos y profesionalizadores que ofrece el enfoque por competencias, y las posibilidades de innovación y renovación que brinda, aspecto, este último, también subrayado por los expertos en Pedagogía en la investigación de López et al. (2016). Sin embargo, el profesorado se muestra dividido respecto al carácter prescriptivo e impositivo conferido a las competencias en los planes de estudios y en las guías docentes. Algunos profesores creen que clarifican las directrices a seguir para enfocar la acción docente y favorecen la rendición de cuentas, por lo que lo valoran positivamente. Para otros, en cambio, supone un serio revés que coarta la creatividad docente y dificulta las posibilidades de innovación y mejora. Los que comparten esta última posición son partidarios de que las competencias tengan un carácter más orientativo que prescriptivo y piensan que su concreción debería haber sido resultado de procesos de negociación y deliberación más participativos. Coinciden así con las tesis formuladas por Tran (2013) cuando afirma que los planes de estudios cerrados y sujetos a un alto grado de control dificultan el desarrollo competencial del alumnado y su transición al mundo laboral.

En relación a los motivos expuestos por el profesorado participante para rechazar el enfoque por competencias destacan, especialmente: la falta de apoyos y recursos; la homogeneización que promueve al fijar las mismas competencias para todo el alumnado y la devaluación de los contenidos (saberes). La oposición entre saberes y competencia ha sido ampliamente debatida durante la creación del EEES, sin embargo Perrenoud (2008) la considera injustificada, pues no hay competencias sin saberes. 
En su opinión, el enfoque por competencias, lejos de dar la espalda a los saberes, les confiere una fuerza nueva, un valor añadido, al vincularlos a las prácticas sociales y a las situaciones complejas de la vida. Entiende, no obstante, que esta postura de enfrentamiento está fundamentada porque no es posible desarrollar competencias sin cuestionar la organización de los saberes en disciplinas compartimentadas y reducir el tiempo asignado a la asimilación de saberes. Esta necesidad de trascender la lógica disciplinar y academicista, tan arraigada en la institución universitaria, para favorecer el aprendizaje por competencias del alumnado ha sido recientemente señalada, entre otros, por López et al. (2016).

Estas valoraciones realizadas por los docentes del estudio sobre el enfoque por competencias evidencian que no existe una tendencia clara ni a favor ni en contra de su adopción.

Las competencias que más interesan a los docentes han sido las transversales, ya que consideran que en su incorporación al currículum y su desarrollo está el verdadero reto. Las competencias más señaladas han sido tanto interpersonales, como sistémicas e instrumentales. Específicamente son: trabajo en equipo, aplicación de la teoría a la práctica y expresión oral-escrita y habilidades comunicativas. Todas ellas altamente valoradas en el mundo laboral (Freire, Teijeiro \& Pais, 2011). Las competencias menos aludidas por los docentes en la investigación han sido el compromiso social y el dominio de una lengua extranjera, a pesar de la relevancia otorgada a estos aspectos en los trabajos de Cabrera et al. (2016).

En general, aunque los docentes que han participado en la investigación consideran que se está progresando en el desarrollo de las competencias transversales en la universidad, de sus intervenciones se deduce que no tienen claro cómo incorporarlas al currículum e integrarlas en la práctica formativa y reconocen abiertamente la frustración y dilemas que ello les plantea. También Villarroel y Bruna (2014) han subrayado el desafío y reticencias que suscita, entre el profesorado universitario, la incorporación de estas competencias al currículum académico. En opinión de estas autoras, la incorporación de estas competencias a la educación superior requiere que los docentes se actualicen en metodologías de enseñanza y evaluación, incorporen actividades prácticas donde observen el despliegue de las competencias esperadas en la asignatura, cuenten con mayor tiempo de preparación de clases, construyan evaluaciones auténticas y proporcionen retroalimentación permanente a los estudiantes. Esto implica mayor inversión y recursos por parte de las instituciones educativas (Villarroel \& Bruna, 2014). Esta es una reivindicación constante del profesorado de este estudio que no sólo denuncia la falta de recursos, apoyos y reconocimiento por parte de las universidades, sino que los considera uno de los mayores obstáculos para la incorporación exitosa del enfoque por competencias. Así lo han subrayado también los miembros de las comisiones de garantía de la calidad de distintas titulaciones en el trabajo de Pozo y Bretones (2015). En este estudio se demanda, a los responsables institucionales y gestores educativos: mejorar la dotación de medios y recursos, establecer sistemas de reconocimiento adecuados, optimizar la coordinación de las acciones, potenciar la colaboración y prestar mayor atención a la formación del personal directamente implicado. Lo más decepcionante de este tipo de reivindicaciones es que no sólo no han recibido aún una respuesta satisfactoria por parte de la actual 
política educativa universitaria, sino que parece poco probable que la reciban debido a los constantes recortes presupuestarios a que se ve sometida la educación universitaria. Esta lamentable situación ha llevado, como señalan los docentes del estudio, a confiar el éxito del enfoque por competencias a la buena voluntad del profesorado.

Junto a estas consideraciones, el profesorado de la investigación subraya un segundo escollo para el desarrollo de las competencias transversales: la brecha existente entre el mundo académico y laboral. Esta escisión, en opinión de Perrenoud (2008), es fruto de una división del trabajo que reposa sobre una ficción, por eso invita a la institución educativa y a los educadores a impulsar una visión más global de los aprendizajes que enfatice su utilidad y aplicación en la vida misma. Aunque, como han reconocido Cabrera et al. (2016), el Proceso de Convergencia está sirviendo de detonante para estrechar lazos entre estos dos ámbitos, la superación total del desencuentro continúa siendo un desafío pendiente que dificulta el desarrollo de las competencias transversales (Rutt et al., 2013; Villarroel \& Bruna, 2014). Para salvar esta situación Parris y Saville (2011) proponen, entre otras medidas: promover intercambios de conocimiento entre universidad y empresa, aumentar la presencia de la formación práctica en los planes de estudios, incorporar experiencias prácticas en empresas a la formación universitaria, reforzar los vínculos con el ámbito empresarial, contratar profesorado con experiencia en el ámbito laboral para realizar labores docentes en la universidad, y participar conjuntamente en el diseño de propuestas formativas.

Cambios como estos no sólo requieren compromisos y apoyos instituciones, sino que deben ir acompañados, y así lo reconocen los docentes del estudio, de un cambio de mentalidad en profesorado y estudiantes. Consideran, en este sentido, que el individualismo del alumnado, junto a la falta de implicación en su aprendizaje, son factores que dificultan el aprendizaje de competencias y obstaculizan los esfuerzos realizados para impulsar su incorporación a la educación superior, opinión ésta subrayada también por Tran (2013).

Sin embargo, entre las dificultades más reiteradas por los docentes para la incorporación exitosa del enfoque por competencias figura la falta de formación del profesorado. La escasa atención deparada a la formación docente del profesorado universitario ha colocado a este colectivo en una situación de debilidad para hacer frente a los retos pedagógicos y culturales que supone pasar de un modelo centrado en la enseñanza a un modelo basado en el aprendizaje de competencias. Su adopción demanda de ellos mejorar la formación práctica y establecer vínculos más estrechos con el mundo empresarial (Parris \& Saville, 2011), desplegar nuevas iniciativas metodológicas que refuercen el compromiso del alumnado con su aprendizaje (Navarro et al., 2015; Tran, 2013), poner en marcha nuevos sistemas de evaluación (Cano \& Fernández-Ferrer, 2016; Medina et al., 2015), ... cambios difíciles de afrontar con éxito si no se tiene el compromiso y la cualificación necesarias. Los profesores así lo han manifestado al reconocer su dificultad, por ejemplo, para diferenciar objetivos de competencias, gestionar el trabajo en grupo o llevar a cabo la evaluación de competencias.

En relación a este último aspecto, los docentes del estudio vinculan la dificultad para evaluar competencias, básicamente, a dos cuestiones: su falta de formación y el carácter academicista que aún prevalece en la institución universitaria. La incor- 
poración de este segundo elemento pone de manifiesto la necesidad de abordar el enfoque por competencias en la universidad desde una perspectiva más holística y compleja que ayude a introducir nuevas claves en los estudios y articular respuestas más integrales y mejor coordinadas desde el punto de vista estructural, pedagógico y didáctico. Se trata de adoptar perspectivas más amplias que abarquen tanto el desarrollo de los docentes como el desarrollo organizativo de las estructuras y las culturas universitarias (Euler, 2015).

En relación al impacto del enfoque por competencias, el profesorado, aunque reconocen algunas mejoras (en la retroalimentación ofrecida a los estudiantes, la expresión oral-escrita de los estudiantes, la participación y el trabajo en grupo, y en los resultados académico), tiende a considerar que la repercusión en la práctica formativa universitaria ha sido nula, aproximándose así a las posiciones ya expresadas por autores como Ermenc \& Vujisic-Zivkovic (2015) o Villarroel y Bruna (2014). Sin embargo, esta apreciación no coincide con los resultados mostrados en otros trabajos, como los realizados por Miguel de y Lázaro de (2016) o Villa et al. (2015) que subrayan las mejoras aportadas por el Proceso de Bolonia a la enseñanza universitaria. En este sentido, y dado que no existe unanimidad a la hora de valorar el impacto real del enfoque por competencias en el contexto universitario, sería interesante ampliar el análisis de experiencias exitosas y profundizar en nuevos factores que pudieran estar incidiendo en dicho éxito.

En el caso concreto de nuestro estudio, las numerosas dificultades subrayadas por los participantes y su visión pesimista sobre el impacto del enfoque por competencias en la práctica evidencian que la adopción del nuevo enfoque educativo en el ámbito universitario no está resultando fácil para el profesorado. La incorporación exitosa del enfoque por competencias a la universidad requiere, como se ha puesto de manifiesto en esta investigación, un cambio de mentalidad de profesores y alumnos, una adecuada formación docente, un mayor compromiso institucional que dote de los apoyos y recursos necesarios y adoptar perspectivas más holísticas que permitan atender a los aspectos culturales del cambio. Esta investigación abre así la puerta a la incorporación de nuevos factores y claves en el estudio de la reforma educativa iniciada.

\section{Referencias}

Albert, M.J. (2006). La investigación educativa. Madrid: McGraw Hill.

Cabrera, N., López, M.C. \& Portillo, C. (2016). Las competencias de los graduados y su evaluación desde la perspectiva de los empleadores. Estudios Pedagógicos, 42(3), 69-87. Recuperado de http://mingaonline.uach.cl/pdf/estped/v42n3/art04.pdf

Callejo, J. (2001). El grupo de discusión: introducción a una práctica de investigación. Barcelona: Ariel.

Cano, E. \& Fernández-Ferrer, M. (Eds.). (2016). Evaluación por competencias: la perspectiva de las primeras promociones de graduados en el EEES. Barcelona: Octaedro Editorial.

Euler, D. (2015). Mejorar las competencias docentes del profesorado universitario es necesario, ipero la innovación sostenible requiere algo más!. Educar, 51(1), 149-165. doi: $10.5565 /$ rev/educar.640 
Ermenc, K. S. \& Vujisic-Živkovic, N. (2015). Competence-based approach in the education of pedagogues-comparative view. Sofia: Bulgarian Comparative Education Society.

Freire, MJ., Teijeiro, M. \& Pais, C. (2011). Políticas educativas y empleabilidad: ¿cuáles son las competencias más influyentes?. Archivos Analíticos de políticas educativas, 19(8), 1-24. Recuperado de http://epaa.asu.edu/ojs/article/view/911

Glaser, B. G. \& Strauss, A. L. (1967). The discovery of Grouded Theory. Chicago: Aldine. Gibbs, G. (2012). El análisis de datos cualitativos en investigación cualitativa. Madrid: Morata. Hallinger, P., Heck, R.H. \& Murphy, J. (2014). Teacher evaluation and school improvement: An analysis of the evidence. Educational Assessment, Evaluation and Accountability, 26(1), 5-28. doi: 10.1007/s11092-013-9179-5

López, M.C., Benedito, V. \& León, M. (2016). El enfoque de competencias en la formación universitaria y su impacto en la evaluación. Una aproximación a la perspectiva de un grupo de profesionales expertos en pedagogía. Formación Universitaria 9(4), 11-22. doi: 10.4067/S0718-50062016000400003

Lozano, F. J., Boni, A., Peris, J., \& Hueso, A. (2012). Competencies in higher education: A critical analysis from the capabilities approach. Journal of Philosophy of Education, 46(1), 132-147. doi: 10.1111/j.1467-9752.2011.00839.x

Medina, A., Domínguez, M. C. \& Sánchez, C. (2013). Evaluación de las competencias de los estudiantes: modelos y técnicas para la valoración. Revista de Investigación Educativa, 31(1), 239-255. doi: 10.6018/rie.31.1.157601

Miguel de, R. \& Lázaro de, M.L. (2016). Educating geographers in Spain: Geography teaching renewal by implementing the european higher education area. Journal of Geography in Higher Education, 40(2), 267-283. doi: 10.1080/03098265.2016.1139556

Navarro, I., González, C., López, B. \& Botella, P. (2015). Aprendizaje de contenidos académicos y desarrollo de competencias profesionales mediante prácticas didácticas centradas en el trabajo cooperativo y relaciones multidisciplinares. Revista de Investigación Educativa, 33(1), 99-117. doi:10.6018/rie.33.1.183971

Parris, M. \& Saville, K. (2011). Piecing together the puzzle of graduate employment: Factors that shape the graduate work expectations of human resource management students. Industry and Higher Education, 25(1), 15-24. doi: 10.5367/ihe.2011.0024

Perrenoud, P. (2008). Construir las Competencias, ¿Es darle la espalda a los saberes?, RedU. Revista de Docencia Universitaria, 6(2), 1-16. Recuperado de http://www. urosario.edu.co/CGTIC/Documentos/construir_competencias_saberes.pdf

Pozo, C. \& Bretones, B. (2015). Dificultades y retos en la implantación de los títulos de grado en las universidades españolas. Revista de Educación, 267, 147-172. doi: 10.4438/1988-592X-RE-2015-367-286

Rutt, L.; Gray, C.; Turner, R.; Swain, J.; Hulme, S. \& Pomero, R. (2013). A social constructivist approach to introducing skills for employment to foundation degree students. Research in Post-Compulsory Education, 18(3), 280-296. doi: 10.1080/13596748.2013.819268

Strauss, A. \& Corbin, J. (2002). Bases de la investigación cualitativa. Técnicas y procedimientos para desarrollar la teoría fundamentada. Colombia: Editorial Universidad de Antioquía.

Tran, T.T. (2013). Limitation on the development of skills in higher education in Vietnam. Higher Education: The International Journal of Higher Education and Educational Planning, 65(5), 631-644. doi: 10.1007/s10734-012-9567-7 
Villa, A., Arranz, S., Campo, L. \& Villa, O. (2015). Percepción del profesorado y responsables académicos sobre el proceso de implantación del Espacio Europeo de Educación Superior en diversas titulaciones de educación. Profesorado. Revista de currículum y formación del profesorado, 19(2), 245-264. Recuperado de http://www. ugr.es/ recfpro/rev192COL1.pdf

Villarroel, V. \& Bruna, D. (2014). Reflexiones en torno a las competencias genéricas en educación superior: Un desafío pendiente. Psicoperspectivas, 13(1), 23-34. doi: 10.5027/PSICOPERSPECTIVAS-VOL13SSUE1-FULLTEXT-335

Watson, R. (2011). A rationale for the development of an extracurricular employability award at a British University. Research in Post-Compulsory Education, 16(3), 371-384. doi: 10.1080/13596748.2011.602248

Fecha de recepción: 12 de diciembre de 2017

Fecha de revisión: 31 de diciembre de 2017

Fecha de aceptación: 6 de marzo de 2018 
